\title{
Optical frequency comb generation using annealing-free Si3N4 films for front-end monolithic integration with Si photonics
}

El Dirani, Houssein; Kamel, Ayman; Casale, Marco; Kerdiles, Sebastien; Monat, Christelle; Letartre, Xavier; Pu, Minhao; Oxenløwe, Leif Katsuo; Yvind, Kresten; Sciancalepore, Corrado

Published in:

Integrated Optics: Devices, Materials, and Technologies XXIII

Link to article, DOI:

10.1117/12.2508565

Publication date:

2019

Document Version

Publisher's PDF, also known as Version of record

Link back to DTU Orbit

Citation (APA):

El Dirani, H., Kamel, A., Casale, M., Kerdiles, S., Monat, C., Letartre, X., Pu, M., Oxenløwe, L. K., Yvind, K., \& Sciancalepore, C. (2019). Optical frequency comb generation using annealing-free $\mathrm{Si}_{3} \mathrm{~N}_{\text {films for front-end }}$ monolithic integration with Si photonics. In S. M. G-B., \& P. C. (Eds.), Integrated Optics: Devices, Materials, and Technologies XXIII (Vol. 10921). [1092108] SPIE - International Society for Optical Engineering. Proceedings of SPIE - The International Society for Optical Engineering https://doi.org/10.1117/12.2508565

\section{General rights}

Copyright and moral rights for the publications made accessible in the public portal are retained by the authors and/or other copyright owners and it is a condition of accessing publications that users recognise and abide by the legal requirements associated with these rights.

- Users may download and print one copy of any publication from the public portal for the purpose of private study or research.

- You may not further distribute the material or use it for any profit-making activity or commercial gain

- You may freely distribute the URL identifying the publication in the public portal 


\section{Optical frequency comb generation using annealing-free Si3N4 films for front-end monolithic integration with Si photonics}

Houssein El Dirani, Ayman Kamel, Marco Casale, Sébastien Kerdiles, Christelle Monat, et al.

Houssein El Dirani, Ayman Kamel, Marco Casale, Sébastien Kerdiles, Christelle Monat, Xavier Letartre, Minhao Pu, Leif Katsuo Oxenløwe, Kresten Yvind, Corrado Sciancalepore, "Optical frequency comb generation using annealing-free Si3N4 films for front-end monolithic integration with $\mathrm{Si}$ photonics," Proc. SPIE 10921, Integrated Optics: Devices, Materials, and Technologies XXIII, 1092108 (4 March 2019); doi: 10.1117/12.2508565 


\title{
Optical frequency comb generation using annealing-free $\mathrm{Si}_{3} \mathrm{~N}_{4}$ films for front-end monolithic integration with Si photonics
}

\author{
Houssein El Dirani ${ }^{1}$, Ayman Kamel ${ }^{2}$, Marco Casale ${ }^{1}$, Sébastien Kerdiles ${ }^{1}$, Christelle Monat ${ }^{3}$, Xavier \\ Letartre $^{3}$, Minhao $\mathrm{Pu}^{2}$, Leif Katsuo Oxenløwe ${ }^{2}$, Kresten Yvind ${ }^{2}$, and Corrado Sciancalepore ${ }^{1}$ \\ 1- CEA-LETI, Minatec, CEA-Grenoble, F-38054, Grenoble, France \\ 2- Technical University of Denmark, Lyngby, Denmark \\ 3- CNRS, Institut des nanotechnologies de Lyon, Lyon, France
}

\begin{abstract}
In this communication, we report on the design, fabrication and testing of silicon-nitride-in-insulator (SiNOI) nonlinear photonic circuits for comb generation in silicon photonics and optoelectronics. The low two-photon absorption when compared with crystalline silicon makes the SiNOI an attractive platform for frequency comb generation. Kerr combs have been recently used in terabit per second coherent communications demos. Such devices can overcome the intrinsic limitations of nowadays silicon photonics notably concerning the heterogenous integration of III-V on SOI lasers for both datacom and telecom applications. By using monolithically-integrated SiN-based Kerr frequency combs, the generation of tens or even hundreds of new optical frequencies can be obtained in dispersion tailored waveguides and resonators, thus providing an all-optical alternative to the heterointegration of hundreds of standalone III-V on Si lasers. However, in all the previous SiNOI-based frequency combs, the silicon nitride film is annealed under long and high temperature which made the cointegration with silicon based optoelectronics elusive. The annealing steps used in common $\mathrm{SiN}$ fabrication processes are not only incompatible with the front-end of line complementary metal-oxidesemiconductor processes, but also costly and long and thus an important cost factor in non-CMOS compatible processes. In our work, we present the fabrication and testing of an annealing-free and crack-free SiNOI. Notably, a 800-nmspanning (1300-2100 nm) frequency comb is generated using 740-nm-thick silicon nitride featuring full compatibility with silicon photonics integrated circuits. This work constitutes a new, decisive step toward time-stable power-efficient Kerr-based broadband sources featuring full process compatibility with Si photonic integrated circuits (Si-PICs) on CMOS-lines.
\end{abstract}

Keywords: Complementary metal-oxide-semiconductor (CMOS), nonlinear integrated optics, Kerr-based comb generation, resonators, photonic integrated circuits (PICs), silicon nitride $\left(\mathrm{Si}_{3} \mathrm{~N}_{4}\right)$.

\section{INTRODUCTION}

Kerr frequency combs constitute a paradigm shift in the development of high-capacity data transmission, integrated spectroscopy, high precision metrology, and frequency synthesis [1]. Since 2010, silicon-nitride-on-insulator (SiNOI) has imposed as an attractive chip-based platform for the generation of wideband frequency combs pumped at telecom wavelengths, because of its relatively high nonlinearity ( $\times 10$ that of silica and larger than that of highly nonlinear Hydex glass [2]) as well as the absence of two-photon absorption and free carrier generation that plague crystalline silicon. In the meanwhile, silicon photonics integrated circuits (Si-PICs) have demonstrated increasing maturity levels for a wide range of optical functions such as III-V-on-Si integrated lasers [3], high-speed modulators [4], Ge-on-Si photodiodes [5], as well as filters and wavelength (de)multiplexers [6], thus continuously highlighting the potential of silicon optoelectronics integration with cost-effective complementary metal-oxide-semiconductor (CMOS) technology [7,8]. In this context, as presented in fig. 1 the monolithic co-integration of Kerr-based frequency combs with Si photonics holds the promise for on-chip high-capacity transmitters that would benefit from the maturity and low cost of CMOS manufacturing and scalability.

Integrated Optics: Devices, Materials, and Technologies XXIII, edited by Sonia M. García-Blanco, Pavel Cheben, Proc. of SPIE Vol. 10921, 1092108 - @ 2019 SPIE - CCC code: 0277-786X/19/\$18 · doi: 10.1117/12.2508565 
The realization of relatively thick (> $700 \mathrm{~nm})$ stoichiometric Si3N4 films, as required by microring frequency combs, which imply both a tight confinement of light and anomalous group velocity dispersion (GVD), remains challenging. In particular, all prior works strictly made use of long high-temperature annealing $\left(\sim 1200{ }^{\circ} \mathrm{C}\right.$ for at least $\left.3 \mathrm{~h}\right)$ of the deposited silicon nitride film [9-12]. This extreme annealing step has been accounted for by the need to densify the silicon nitride film through driving out excess hydrogen and break N-H bonds, so as to get closer to a stoichiometric Si3N4 film and reduce the material absorption loss in the C-band. However, this annealing induces thermal stress that eventually leads to cracks during the device processing unless sophisticated pre-patterning strategies are adopted prior to the film deposition $[10,11]$.

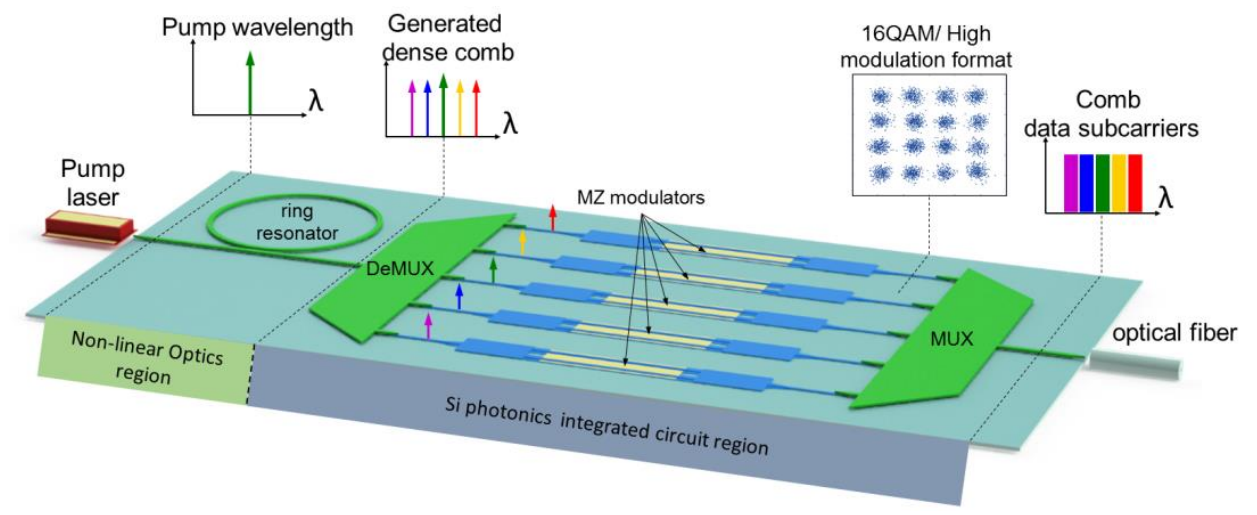

Fig. 1. Principles of high transfer rate communications with Kerr frequency combs. Artist's view of a future high transfer rate transmitter, leveraging a Kerr frequency comb source. DeMUX, de-multiplexer; MZ-modulators, Mach-Zehnder modulator; MUX, multiplexer.

Yet, in the context of nonlinear optics-silicon optoelectronics co-integration, these extreme annealing temperatures would severely degrade the front-end silicon optoelectronics circuit underneath. Specifically, doped optical circuits would be unacceptably affected by the undesirable dopant diffusion in junction-based Si modulators and by the heterointerface degradation of Ge-on-Si photodetectors. Very recently, we reported a new method that avoids thermal annealing for realizing relatively thick $(740 \mathrm{~nm})$ crack-free Si3N4-based straight nanowaveguides with good linear and nonlinear properties measured by self-phase modulation [15].

\section{NONLINEAR SI3N4 CIRCUITS FABRICATION}

Here, we report for the first time the realization of annealing-free silicon nitride comb microresonators, following a tailored deposition method which minimizes the hydrogen content. Our annealing-free and crack-free fabrication process (shown in Fig. 2) provides our devices with the right specification (microring GVD and characteristics) to underpin Kerr frequency combs, thus representing a significant step toward the full compatibility of Si3N4-based Kerr comb sources with the thermal budgets of Si photonics processing. In contrast to all previous approaches, our process does not exceed neither the dopant activation temperature $\left(1030^{\circ} \mathrm{C}\right)$ required for Si modulators [13], nor the $\mathrm{H} 2$ annealing thermal budget used for dislocations control for Ge-on-Si photodiodes $\left(825^{\circ} \mathrm{C}\right)[14]$.

In order to control strain and to prevent cracks from appearing, the silicon nitride layer is deposited on a (non-patterned) substrate via low-pressure chemical vapor deposition (LPCVD) in two steps of 370-nm-thick layer each. The deposition is carried out with a tailored ultra-low deposition rate $(\sim 2 \mathrm{~nm} / \mathrm{min})$ to produce a very high quality film, which is denser optically, and notably offering a higher nonlinear index $(\mathrm{n} 2=3.6 \times 10-19 \mathrm{~m} 2 \cdot \mathrm{W}-1)$ [15].

Critically, under such low deposition rates, the thermal activation energy enables silicon and nitrogen to dispose at the nitride film surface via atomic surface migration phenomena, while compelling hydrogen to escape the film. Furthermore, between the two deposition stages, the wafer is rotated by $45^{\circ}$ in order to distribute the uniaxial strain along the overall film thickness, thus avoiding film cracks upon subsequent subtractive patterning. Each deposition run is carried out at $780{ }^{\circ} \mathrm{C}$ with post-deposition cooling to around $630{ }^{\circ} \mathrm{C}$ for 20 minutes. Controlled ramp-ups and -downs from/to $780{ }^{\circ} \mathrm{C}$ at $10^{\circ} \mathrm{C} /$ minute to/from $630^{\circ} \mathrm{C}$ are used prior to each deposition which is carried out under a $112 \mathrm{mTorr}$ pressure using $\mathrm{NH} 3(200 \mathrm{sccm})$ and $\mathrm{SiH} 2 \mathrm{Cl} 2(80 \mathrm{sccm})$ as precursor gases. 
(a) Substrate Thermal oxydation

(b) First Ultra-low SiN deposition

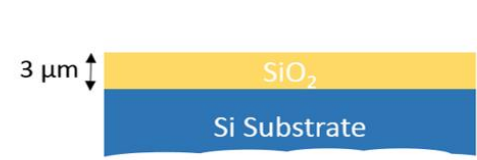

(c) Cooling at $630^{\circ} \mathrm{C}$

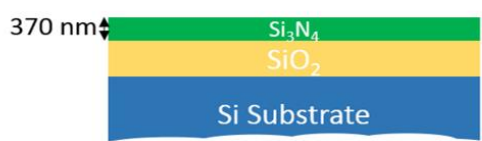

(e) Dry etch and resist stripping

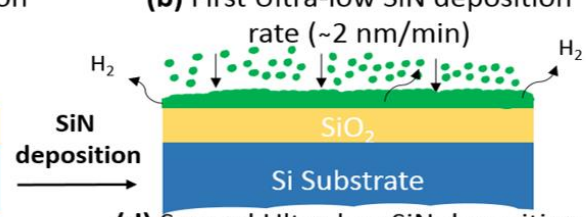

(d) Second Ultra-low SiN deposition

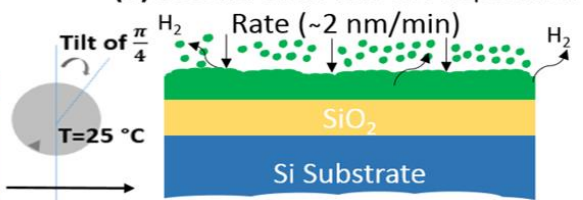

(f) High-density plasma silica deposition

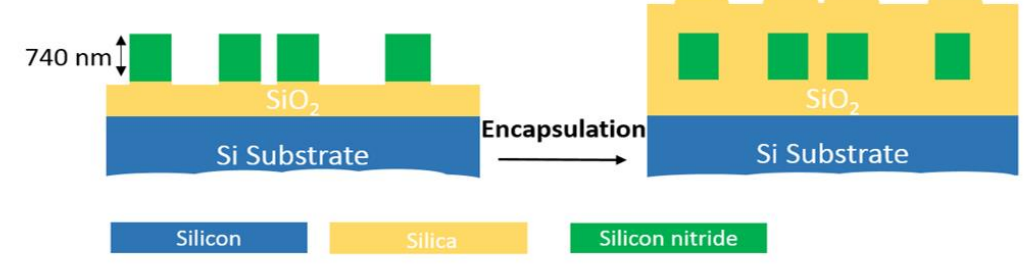

Fig. 2. Schematics of the annealing-free fabrication process for Si3N4 nonlinear photonics (a)-(f).

By measuring the wafer bow, before and after removing the silicon nitride from the wafer back side, the material morphological characterization revealed a tensile strain around +1200 MPa. Such high tensile strain is a clear indication of the stoichiometry of the material (i.e., minimization of residual hydrogen content) which is higher than annealed silicon nitrides presented in prior works [11].

The Si3N4 circuits were patterned through a combination of deep UV lithography (I-line, 780-nm-thick resist) and fluoride-based (CF4-CH2F2-O2) dry etching. They were encapsulated by $3-\mu \mathrm{m}$-thick $\mathrm{SiO} 2$ cladding layer at $400{ }^{\circ} \mathrm{C}$ using high-density plasma-enhanced chemical vapor deposition (HDP-PECVD) to avoid void formation, while a Boschlike process was used to perform the deep etch for lateral facets patterning. Figs. 3(a), and 3(b) show optical and electron scanning microscope images of the fabricated devices.
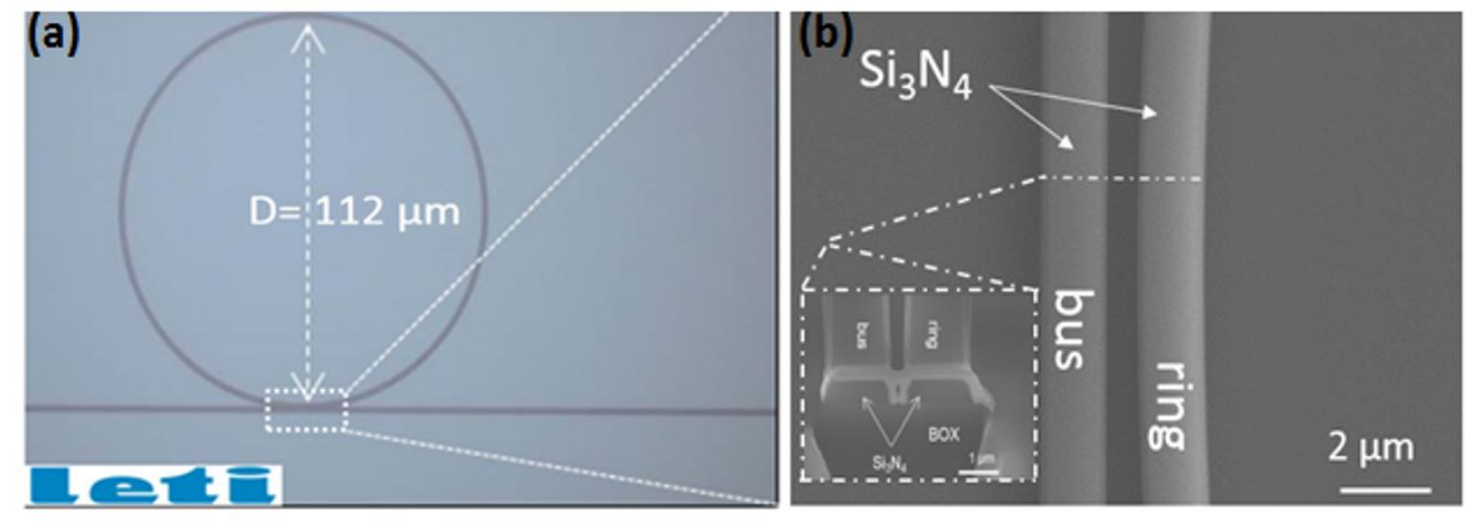

Fig. 3. Optical microscope (a) and scanning electron microscope (c) images of the ring and coupling region, respectively. Inset: Crosssection of the void-free coupling gap 


\section{LINEAR AND NONLINEAR OPTICAL CHARACTERIZATION}

The measured spectrum of an annealing-free silicon-nitride-on-insulator microring with a 56- $\mu$ m radius is shown in Fig. 4. A native line spacing frequency comb spanning across about $730 \mathrm{~nm}$ between $1340 \mathrm{~nm}-2070 \mathrm{~nm}$ was measured when a continuous-wave pump power of $\sim 1 \mathrm{~W}$ at $1569 \mathrm{~nm}$ - was coupled in the bus waveguide. The loaded quality factor of the ring resonator separated by a $350 \mathrm{~nm}$ gap from the bus waveguide exceeds 580,000 at the pump wavelength. The cross-section dimensions $(1.5-\mu \mathrm{m}$-wide $\times 740$-nm-thick) of the ring ensure that GVD is anomalous at the pump wavelength.

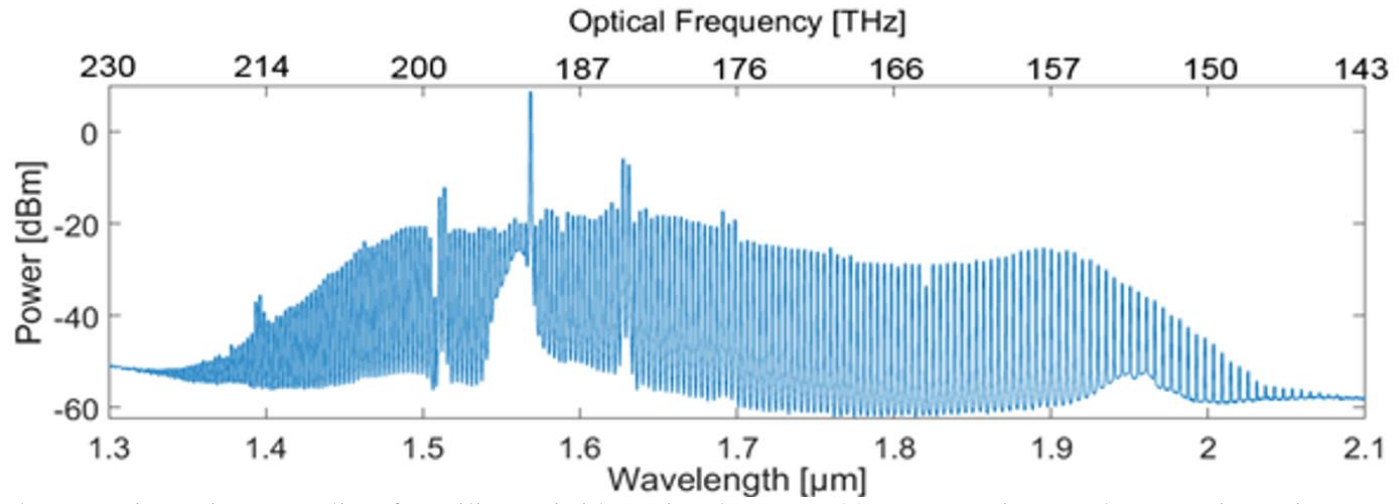

Fig. 4. Comb generation using annealing-free silicon nitride on insulator. A 730-nm-spanning comb generation using a 56- $\mu \mathrm{m}$-radius Si3N4 microresonator.

Interestingly, a slight signature of residual hydrogen-related absorption can be observed in the comb around $1508 \mathrm{~nm}$, but it remains comparable to previous works employing film annealing and does not hinder the generation of a relatively wide and flat comb spectrum.

Another indication of the remaining $\mathrm{N}-\mathrm{H}$ bonds is provided by the spectral dependence of the intrinsic quality factor $\left(Q_{i}\right)$ measured for a $\mathrm{Si}_{3} \mathrm{~N}_{4}$ micro-ring resonator with $1.4 \mu \mathrm{m}$ waveguide width (see figure 5 ). It roughly increases by a factor 2 while moving away from the N-H overtone absorption peak (near $1520 \mathrm{~nm}$ ), showing the presence of residual N-H bonds in our film. The losses in the ring can be estimated by the expression [16] $\alpha=\frac{k_{0} n_{g}}{Q_{i}}$ where $k_{0}$ is the wavenumber and $n_{g}$ is the mode group index of the waveguide with similar width. The difference between the losses at $1550 \mathrm{~nm}\left(Q_{i}=350,000\right)$ and the losses at $1520 \mathrm{~nm}\left(Q_{i}=190,000\right)$ can thus be estimated to be $0.9 \mathrm{~dB} / \mathrm{cm}$. This additional loss due to residual N-H absorption for our annealing-free process is comparable to the value $(0.6 \mathrm{~dB} / \mathrm{cm})$ inferred for high-temperature annealed $\mathrm{Si}_{3} \mathrm{~N}_{4}$ waveguides [17], and, as shown below, it does not preclude the oscillation and comb generation in the C-band.

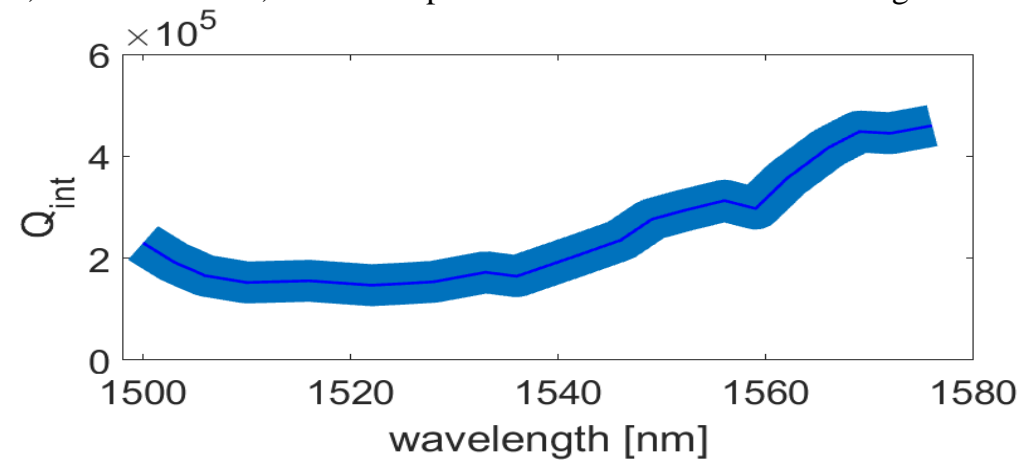

Fig. 5.Intrinsic quality factor as a function of wavelength for an annealing-free silicon nitride microring resonator (waveguide width equal to $1.4 \mu \mathrm{m}$ ). The darker colored line shows the mean value and the brighter shadowed areas illustrates the standard deviation of the measurements.

The dispersion of our devices is measured by scanning an ECDL light while recording the device transmission and two calibration traces from a fiber cavity and a high-finesse free space cavity with FSR of $171 \mathrm{MHz}$ and $175 \mathrm{MHz}$, respectively. 
$D_{\mathrm{int}}(\mu)=\omega_{\mu}-D_{1} \mu=\frac{D_{2}}{2} \mu^{2}+\frac{D_{3}}{6} \mu^{3}+\ldots$ is the integrated dispersion where $\omega_{\mu}$ is the angular frequency, $\mu$ is the mode number relative to the pumped mode and $D_{i}$ are the dispersion coefficients. The measured dispersion is showed in figure 6 . A fit of the measured dispersion gives an anomalous dispersion value of $D_{2} / 2 \pi=511 \mathrm{kHz}$ at $1580 \mathrm{~nm}$ which is equivalent to a group velocity dispersion of $29 \mathrm{ps} / \mathrm{nm} / \mathrm{km}$. This value compares well with our finite element modeling of the dispersion which gave us an anomalous dispersion estimate of $50 \mathrm{ps} / \mathrm{nm} / \mathrm{km}$ at the same wavelength. The values of simulated and measured dispersion are in agreement with the simulated dispersion of similar annealed Si3N4 waveguides presented in [2].

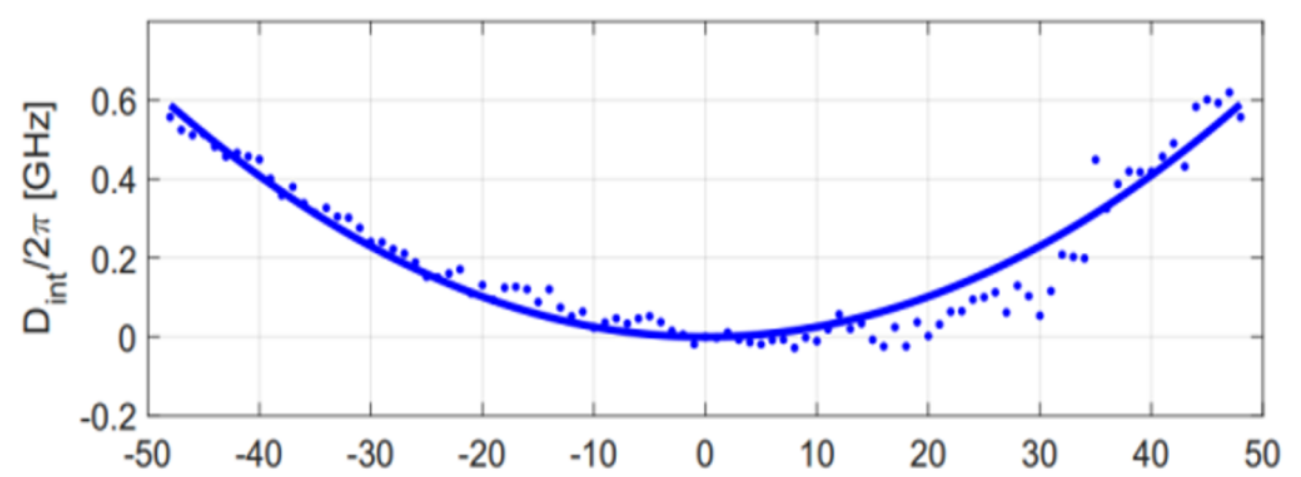

Fig. 6. Dispersion measurement of the TE mode of a ring resonator with $200 \mu$ m radius and $1.5 \mu$ m width

In order to measure the threshold power for optical parametric oscillation (OPO), the device was pumped with increasing power levels coupled to the bus waveguide. The laser wavelength was scanned across a resonance at $1570 \mathrm{~nm}$. The chip output light was passed through a short pass filter with a cut-off wavelength of $1560 \mathrm{~nm}$ that blocks the pump light with an extinction ratio of $60 \mathrm{~dB}$ and was then detected by a photodiode. The peak power of the filtered short wavelength OPO signal at each power coupled to the bus waveguide shows that the measured signal power is plotted in Fig. 7, showing a threshold power of $\sim 83 \mathrm{~mW}$.

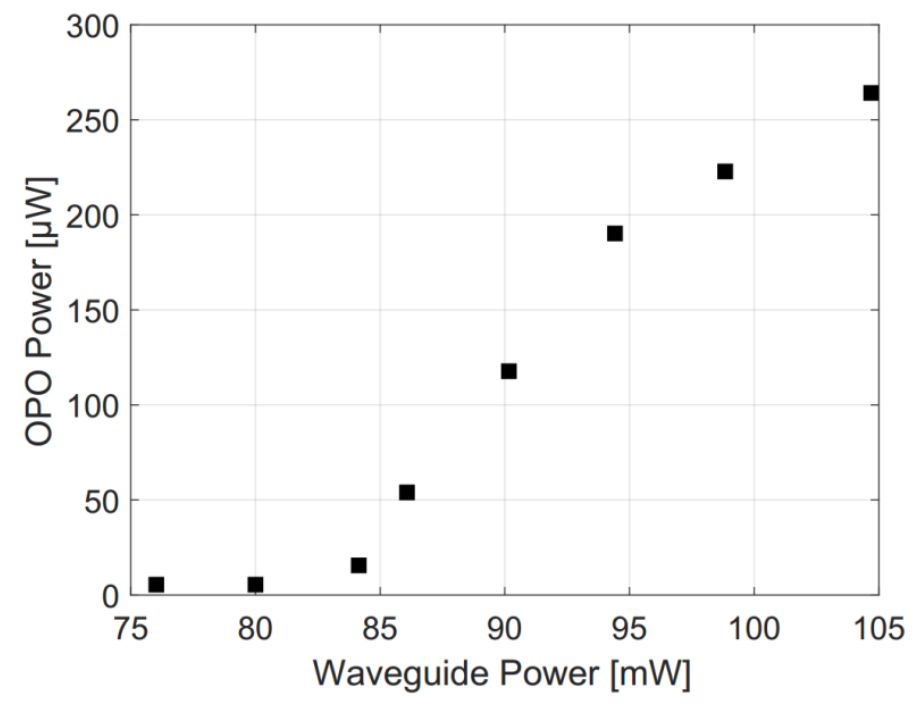

Fig. 7. Plot of short-wavelength OPO power with input power in the bus waveguide. 


\section{CONCLUSIONS}

In conclusion, generating a wideband comb at telecom wavelengths using annealing-free silicon nitride nonlinear circuits featuring a full FEOL process compatibility with Si photonics is possible [18]. Via such demonstration, we claim the first-time realization of annealing-free silicon nitride frequency comb microresonators, following a tailored deposition method, minimizing the hydrogen content. The right specification (microring group velocity dispersion and characteristics) are provided by our annealing-free and crack-free fabrication process to underpin Kerr frequency combs, thus representing a significant step toward the full compatibility of $\mathrm{Si}_{3} \mathrm{~N}_{4}$-based Kerr-comb sources monolithic integration with standard CMOS and Si photonics processing. Through allowing the monolithic integration of broadband comb sources with CMOS-compatible optoelectronics, our work represents a milestone toward the realization of nextgeneration Petabit/s data transmitters on a chip.

Funding. DOPT 2020 internal funding program (CEA-LETI). Danish Research Council SPOC (DNRF-123) center of excellence silicon photonics for communications (SPOC) (DTU). ERC H2020 GRAPHICS (648546) and the Institut Universitaire de France (INL).

\section{REFERENCES}

[1] Marin-Palomo, P., Kemal, J. N., Karpov, M., Kordts, A., Pfeifle, J., Pfeiffer, M. H. and Rosenberger, R., "Microresonator-based solitons for massively parallel coherent optical communications," Nat. Papers 546(7657), 274 (2017).

[2] Moss, D. J., Morandotti, R., Gaeta, A. L. and Lipson, M., "New CMOS-compatible platforms based on silicon nitride and Hydex for nonlinear optics," Nat. Phot. Papers 7(8), 597 (2013).

[3] Bakir, B. B., Descos, A., Olivier, N., Bordel, D., Grosse, P., Augendre, E. and Fedeli, J. M., "Electrically driven hybrid Si/III-V Fabry-Pérot lasers based on adiabatic mode transformers," Opt. Exp. Papers 19(11), 10317-10325 (2011).

[4] Liao, L., Samara-Rubio, D., Morse, M., Liu, A., Hodge, D., Rubin, D. and Franck, T., "High speed silicon MachZehnder modulator,' Opt. Exp. Papers 13(8), 3129-3135 (2005).

[5] Vivien, L., Rouvière, M., Fédéli, J. M., Marris-Morini, D., Damlencourt, J. F., Mangeney, J. and Pascal, D., "High speed and high responsivity germanium photodetector integrated in a Silicon-On-Insulator microwaveguide," Opt. Exp. Papers 15(15), 9843-9848 (2007).

[6] Bogaerts, W., Selvaraja, S. K., Dumon, P., Brouckaert, J., De Vos, K., Van Thourhout, D. and Baets, R., "Silicon-oninsulator spectral filters fabricated with CMOS technology," IEEE. J. Sel. Topics in quant. Elec., Papers 16(1), 33-44 (2010).

[7] Reed, G. T., "Device physics: the optical age of silicon,” Nat. Papers 427(6975), 595 (2004).

[8] Asghari, M. and Krishnamoorthy, A. V., "Silicon photonics: Energy-efficient communication," Nat. Phot. Papers 5(5), 268 (2011).

[9] Levy, J. S., Gondarenko, A., Foster, M. A., Turner-Foster, A. C., Gaeta, A. L. and Lipson, M., "CMOS-compatible multiple-wavelength oscillator for on-chip optical interconnects," Nat. Phot. Papers 4(1), 37 (2010).

[10] Luke, K., Dutt, A., Poitras, C. B. and Lipson, M., "Overcoming Si3N4 film stress limitations for high quality factor ring resonators," Opt. Exp. Papers 21(19), 22829-22833 (2013).

[11] Pfeiffer, M. H., Kordts, A., Brasch, V., Zervas, M., Geiselmann, M., Jost, J. D. and Kippenberg, T. J., "Photonic Damascene process for integrated high-Q microresonator based nonlinear photonics, "' Optica, Papers 3(1), $20-25$ (2016). 
[12] Kim, S., Han, K., Wang, C., Jaramillo-Villegas, J. A., Xue, X., Bao, C. and Qi, M., "Dispersion engineering and frequency comb generation in thin silicon nitride concentric microresonators," Nat. Com. Papers 8(1), 372 (2017).

[13] Streshinsky, M., Ding, R., Liu, Y., Novack, A., Yang, Y., Ma, Y. and Baehr-Jones, T., “ Low power 50 Gb/s silicon traveling wave Mach-Zehnder modulator near 1300 nm ," Opt. Exp. Papers 21(25), 30350-30357 (2013).

[14] Michel, J., Liu, J. and Kimerling, L. C. "High-performance Ge-on-Si photodetectors," Nat. Phot. Papers 4(8), 527 (2010).

[15] El Dirani, H., Casale, M., Kerdiles, S., Socquet-Clerc, C., Letartre, X., Monat, C. and Sciancalepore, C., "CrackFree Silicon-Nitride-on-Insulator Nonlinear Circuits for Continuum Generation in the C-Band,” IEEE Phot. Tech. Lett. Papers 30(4), 355-358 (2018).

[16] J. S. Levy, Ph.D. thesis, Cornell University, New York, 2011.

[17] Krückel, C. J., Fülöp, A., Ye, Z. and Andrekson, P. A., “ Optical bandgap engineering in nonlinear silicon nitride waveguides," Opt. Exp. Papers 25(13), 15370-15380 (2017).

[18] El Dirani, H., Kamel, A., Casale, M., Kerdiles, S., Monat, C., Letartre, X. and Sciancalepore, C. "Annealing-free Si3N4 frequency combs for monolithic integration with Si photonics,” Appl. Phys. Lett. Papers 113(8), 081102-081105 (2018). 